\title{
ENGENHARIA DO CONHECIMENTO DE SISTEMAS DE INFORMAÇÃO EM SAÚDE COM BASE EM MODELOS MULTINIVEL MINIMALISTAS
}

Knowledge engineering of healthcare information systems based on minimalist multilevel models

\author{
Luciana Tricai Cavalini'; Timothy Wayne Cook ${ }^{2}$
}

Resumo A manutenção em longo prazo dos registros eletrônicos de saúde dentro de seu contexto original de informação é uma exigência ética que conflita com a necessidade constante de migração das informações para novos sistemas, quando eles são desenvolvidos e melhorados. As especificidades de cada estabelecimento de saúde em particular excluem a viabilidade de um registro de saúde monolítico, e garantir a interoperabilidade entre os sistemas é o principal desafio enfrentado pelos pesquisadores da área de informática em saúde. Diferentes abordagens de modelagem multinível têm sido desenvolvidas para lidar com essa complexidade. No entanto, as especificações originais de modelagem multinível são direcionadas para o desenvolvimento de prontuários eletrônicos de base hospitalar, o que dificulta sua adoção para o desenvolvimento de aplicativos mais simples, com propósitos específicos, para uso em situações de saúde extra-hospitalares. Este artigo apresenta a engenharia do conhecimento de um modelo multinível minimalista que pode ser implementado por desenvolvedores para todo o espectro mais amplo de aplicações na área de saúde. Ao utilizar padrões da indústria de tecnologia da informação, esta abordagem permite uma maior adoção de tecnologias interoperáveis em saúde.

Palavras-chave: Registros Eletrônicos de Saúde, Gestão do Conhecimento, Design de Programas Computacionais.

Abstract In The long-term maintenance of electronic health records within their original context of information is an ethical requirement that conflicts with the constant need to migrate the information into new systems as they are developed and improved. The specifics of every particular healthcare setting preclude the feasibility of a monolithic health record, and the achievement of interoperability between systems is the primary challenge faced by health informatics researchers. Different multilevel modeling approaches have been studied to deal with this complexity. Nevertheless, the original multilevel modeling specifications are targeted to the development of hospital-based electronic medical records, which adds complexity to the development of simpler purposespecific applications for extra-hospital healthcare situations. This paper presents the knowledge engineering of a minimalist multilevel model that can be implemented by developers across the broader spectrum of healthcare applications. By using industry standard technologies, this approach enables the wider adoption of interoperable technology for healthcare.

Keywords: Electronic Health Records, Knowledge Management, Software Design.

1. Professora Adjunta do Departamento de Tecnologias da Informação e Educação em Saúde da Faculdade de Ciências Médicas da UERJ - Doutorado em Saúde Coletiva pelo Instituto de Medicina Social da UERJ; 2. Colaborador Internacional do Laboratório “Multilevel Healthcare Information Modeling" (LA-MLHIM), Associado ao Instituto Nacional de Ciência e Tecnologia - Medicina Assistida por Computação Científica - Mestre em Health Informatics pela University of Central Lancashire. 


\section{Introdução}

A efetividade dos sistemas de saúde pode ser medida pela sua adequação ao perfil demográfico e epidemiológico da população-alvo. ${ }^{1}$ Nas últimas décadas, esses perfis têm apresentado mudanças rápidas e complexas, devidas à globalização, como pode ser visto durante a ocorrência de epidemias e pandemias, ${ }^{2}$ bem como no dia a dia superlotado dos serviços de emergência. ${ }^{3} \mathrm{O}$ tempo necessário para responder a essas mudanças pode se estender por semanas ou até mais, com a correspondente falta de eficácia das intervenções, enquanto analistas coletam dados de sistemas de informação incompatíveis ou de registros em papel. ${ }^{4}$ A incorporação da tecnologia da informação na área da saúde tem sido proposta como uma estratégia para superar a situação atual, mas existem obstáculos para a realização desta promessa, que são derivados das significativas complexidades da informação em saúde nas dimensões de espaço, tempo e ontologia. ${ }^{5}$

Em relação à dimensão espacial, há especificidades relacionadas em relação tanto à população quanto aos serviços de saúde no nível local. Dada uma grande área geográfica (por exemplo, uma área metropolitana), composta por um conjunto de áreas menores (por exemplo, municípios), as áreas menores apresentam heterogeneidade em termos de seus perfis demográficos e epidemiológicos, dos determinantes sociais da saúde e dos níveis de acesso à saúde. Assim, áreas próximas ou adjacentes podem apresentar diferentes necessidades de saúde. Dependendo das configurações do sistema de saúde do nível nacional para o nível local, a resposta política a essas necessidades de saúde pode variar de uma área menor para outra, gerando uma complexidade adicional. ${ }^{6}$

A complexidade temporal é associada à evolução tecnológica na área da saúde, que por sua vez se relaciona com o desenvolvimento de equipamentos e técnicas de diagnóstico, de produtos farmacêuticos e de outras tera- pias. Assim, o desenvolvimento tecnológico na medicina cria novos conceitos e altera os previamente existentes, o que resulta em mudanças constantes na prática médica. ${ }^{7}$

A complexidade ontológica é parcialmente derivada da dinâmica temporal. No entanto, existe um problema específico, derivado do fato de que a ciência médica está localizada na interface entre as ciências biológicas, sociais e exatas. Diferentes definições de um único conceito de saúde podem coexistir; assim, a probabilidade de se chegar a um consenso em relação a qualquer conceito na área da saúde é baixo. A variabilidade de definições conceituais entre escolas médicas, combinada com a heterogeneidade das políticas de saúde e das culturas médicas, pode diminuir mais ainda o nível de consenso sobre conceitos médicos. ${ }^{8}$

É ainda importante notar que cada provedor de saúde tem diferentes necessidades de informação. Portanto, os aplicativos e as interfaces de usuário precisam ser muito específicos para garantir usabilidade, ${ }^{9}$ e há evidências de que grandes sistemas padronizados são incapazes de se adaptar às rápidas mudanças típicas das novas tecnologias médicas emergentes. ${ }^{10}$ Assim, os sistemas de informação de saúde da atualidade não são interoperáveis e têm altos custos de manutenção, a menos que os padrões e as terminologias evoluam rapidamente, o que é pouco factível. Em consequência, a saúde é o serviço menos informatizado, quando comparado a outros setores da economia global. ${ }^{11}$

Muitas soluções diferentes (e muito dispendiosas) têm sido propostas para os desafios de interoperabilidade e manutenção dos registros eletrônicos de saúde, com resultados limitados. ${ }^{10}$ Os esforços mais bem sucedidos neste domínio se referem à adoção de métodos de engenharia de software orientados a modelos, sendo que já existem propostas nacionais sendo executadas neste sentido. 12,13 Mais especificamente, nas últimas duas décadas, uma abordagem diferente tem sido proposta, e é genericamente denominada de Modelagem Multi- 
nível. Sua principal característica é a separação entre os mecanismos de persistência de dados e a engenharia do conhecimento. ${ }^{14}$ Este artigo apresenta os principais aspectos técnicos da engenharia do conhecimento em modelagem multinível de sistemas de informação de saúde, e propõe um modelo multinível de arquitetura minimalista que permite o desenvolvimento de aplicações biomédicas com propósitos específicos, para além dos prontuários eletrônicos de âmbito hospitalar.

\section{Modelagem Multinível}

\section{Princípios}

Os princípios centrais da Modelagem Multinível (MM) baseiam-se na definição de uma estrutura de software orientada a objetos composta por um mínimo de dois níveis. O nível mais baixo é o Modelo de Referência (MR), em que os tipos de dados genéricos e as estruturas de dados são definidos por classes. O segundo nível é o Modelo de Domínio (MD), em que cada conceito específico de um domínio da saúde dado é modelado pela definição de restrições sobre as classes do MR. Em MM, as classes do MR são persistentes e, portanto, tendem a ser estáveis ao longo do tempo, de modo que as mudanças nos mecanismos de persistência são limitadas. No MD, as definições de restrição sobre o MR fornecem a interpretação semântica dos objetos armazenados. Os artefatos de MD são criados e editados por especialistas do domínio da saúde, sem a necessidade de interpretação do conhecimento pelos desenvolvedores de software através de interações ad hoc. A idéia subjacente da MM é que as mudanças nas regras de estrutura e de negócios de um registro eletrônico de saúde são refletidas no MD e não no MR. ${ }^{15}$

Há três especificações MM disponíveis: o modelo dual original proposto pela Fundação openEHR, ${ }^{16}$ a Norma
ISO $13606,{ }^{17}$ e as especificações Multilevel Healthcare Information Modeling (MLHIM). ${ }^{18}$ Apesar da disponibilidade aberta dessas especificações, há poucos registros de implementações de registros baseados em MM em serviços de saúde reais, muitos deles sendo projetos acadêmicos. ${ }^{16,19}$

Uma das razões para isso é a complexidade do modelo openEHR original, que é principalmente concebido para o desenvolvimento de prontuários eletrônicos hospitalares (PEHs). ${ }^{20}$ Os PEHs, no entanto, não são os únicos suportes existentes para informações de saúde, uma vez que o número de dispositivos que coletam dados biomédicos está aumentando rapidamente. ${ }^{21} \mathrm{~A}$ maior parte dos dispositivos médicos não é concebida para suportar os requisitos de processamento de um PEH completo, apesar de que o software embarcado em tais dispositivos deve ser capaz de transmitir os dados recolhidos de forma consistente, utilizando o padrão da indústria. ${ }^{22}$ Assim, faz-se necessário o desenvolvimento de um método para representar os dados em saúde, a partir de uma diversidade de fontes, de uma forma semanticamente coerente, de modo que os dados agregados sobre um indivíduo possam ser analisados desde o nível local até o mais agregado. ${ }^{23}$

\section{Representação do Conhecimento em Modelagem Multinível}

A adoção mais ampla de aplicações baseadas em MM, em cenários reais de saúde, depende da definição de uma arquitetura padrão para a representação de conceitos do domínio. No openEHR e no ISO 13606, a solução foi dada pelo desenvolvimento de uma linguagem específica de domínio (Domain-Specific Language - DSL) para expressar as restrições ao MR em estruturas computacionais denominadas "arquétipos". Em ambos os projetos, esta DSL é a Archetype Definition Language (ADL). ${ }^{24}$ 
A ADL é a escolha teórica ideal para a representação de conhecimento na área da saúde, já que uma DSL é, por definição, a forma mais adequada para representar os conceitos de um domínio específico. Como qualquer $\mathrm{DSL}^{25}$ a ADL permite a representação do conhecimento ao nível exato de abstração do domínio, o que, em princípio, permite que especialistas do domínio da saúde possam desenvolver e validar arquivos em ADL (ou seja, arquétipos). No entanto, os benefícios evidentes da ADL têm sido ultrapassados pelos custos de se educar os especialistas de domínio da saúde para que estes possam usá-la para a modelagem do conhecimento nos projetos openEHR e ISO $13606 .{ }^{26}$ Enquanto esta barreira educacional não for superada, os programadores ainda continuarão responsáveis por escrever, editar e analisar arquivos ADL para a implementação prática de aplicativos baseados em ISO 13606 e openEHR. ${ }^{27}$ Assim, a adoção de uma DSL pura em MM pode representar uma barreira à entrada de programadores e analistas em cenários reais de implementação de larga escala, porque pressupõe a superação da curva de aprendizagem do $A D L$, e essa linguagem não é coberta nos currículos de ciência da computação.

Há também uma característica técnica da ADL que tem consequências para o modelo de governança da engenharia do conhecimento em ISO 13606 e openEHR: a forma de identificação dos arquétipos. Dado que os identificadores dos arquétipos (os archetype IDs) são legíveis, isso pressupõe que exista, obrigatoriamente, uma relação biunívoca entre qualquer conceito clínico e seu arquétipo correspondente, apenas especializações ou versionamentos do mesmo arquétipo sendo permitidos em alguns casos. ${ }^{28} \mathrm{Em}$ consequência, o modelo de governança de arquétipos deve ser de cima para baixo e centralizado. ${ }^{29}$ Esta escolha de um controle centralizado da modelagem do conhecimento pode ser uma das causas para a adoção lenta das especificações openEHR (apesar da inquestionável qualidade técnica das especificações) e, certamente, se choca com a difícil obtenção de consensos, o que é um aspecto intrínseco do conhecimento em saúde. ${ }^{30}$

Ao mesmo tempo em que a ADL foi estabelecida como a DSL para a modelagem de conhecimento nas especificações openEHR, as tecnologias baseadas em eXtensible Markup Language (XML) tornaram-se onipresentes em toda a indústria de informática, sendo a espinha dorsal da emergente Web Semântica. ${ }^{31}$ Assim, há um número crescente de programadores de software que estão familiarizados com o uso de tecnologias XML. ${ }^{32}$ Tecnologias XML foram adotadas como soluções parciais para a normalização de dados de saúde em aplicações baseadas em modelagem tradicional de um nível ${ }^{33}$ e, para sistemas baseados em MM, elas apresentaram um desempenho tão bom quanto a $\mathrm{ADL}^{34}$

Dado que a adoção crescente de MM pode ajudar na superação do desafio da interoperabilidade semântica em saúde, é desejável aumentar o número de desenvolvedores de software baseado em MM. Assim, a adoção de padrões da indústria pode ajudar a garantir a sustentabilidade e melhorar a relação custo-efetividade dos registros eletrônicos em saúde. Com base nesse raciocínio, nas especificações MLHIM, a XML Schema Definition (XSD) foi a linguagem adotada. $\mathrm{O}$ arquivo XSD que define o MD nas especificações MLHIM é denominado Concept Constraint Definition(CCD), cuja finalidade é expressar a combinações e as restrições necessárias e suficientes das classes do MR MLHIM para a representação de um dado conceito de saúde, sendo assim análogo aos arquétipos openEHR e ISO $13606 .{ }^{18}$

O modelo de governança dos CCDs MLHIM é de baixo para cima e descentralizado, alinhado com exigências sócio-políticas globais de multilinguagem e multiculturalidade, uma vez que se permitem quantas implementações de CCD forem necessárias para o mesmo conceito. O conflito entre dois CCDs diferentes para o mesmo conceito é evitada pela atribuição de um 
Universal Unique Identifier (UUID) para cada CCD individual (18). A interoperabilidade semântica dos CCDs é garantida pela validação de CCDs diferentes de acordo com o MR MLHIM através de uma rede semântica distribuída e baseada na Web, denominada Healthcare Knowledge Component Repository (HKCR), um sistema de gerenciamento de conteúdo baseado em Plone que expõe os metadados dos CCDs na Internet via RSSfeeds, proporcionando assim busca humana ou automática de CCDs. Embora seja previsto que, no início, haverá muitos CCDs para um dado conceito, ao passo que a cultura de modelagem do conhecimento amadureça, os melhores CCDs serão mais utilizados. Assim, naturalmente, estes subirão ao topo, como já se viu em muitos casos de estabelecimento de padrões de software livre de facto nas últimas duas décadas. ${ }^{35}$

\section{Modelo Multinível Minimalista}

O Como visto anteriormente, existe uma necessidade de expansão das possibilidades de implementação baseadas em MM para além do tradicional PEH centrado no hospital. Para aplicações biomédicas, há a necessidade de uma forma padronizada de representação dos dados em seu contexto semântico, permitindo a agregação de dados individuais espalhados em múltiplos aplicativos, para a execução de sistemas de apoio à decisão no ponto de cuidado. ${ }^{36}$ Assim, dispositivos médicos móveis, que são geralmente específicos para um propósito, não precisam ser projetados para suportarem os requisitos de processamento de uma plataforma completa de $\mathrm{PEH}$, embora os dados por eles coletados devam ser considerados como parte do registro de saúde completo da pessoa. ${ }^{37}$

Neste sentido, as especificações MLHIM adotam os aspectos positivos de vários padrões de informação de saúde. Assim, em MLHIM, os princípios de MM foram adotados, bem como a arquitetura das especificações. Além disso, tendo em conta que a Norma ISO 13606 define um MR menor, em comparação com o modelo openEHR, incorporando um resíduo semântico menor do que as classes do MR, esta abordagem foi aplicada às especificações MLHIM. No entanto, o MR da ISO 13606 não é abrangente o suficiente para permitir o desenvolvimento de registros eletrônicos de saúde, sendo mais adequado para definir a troca de mensagens entre os sistemas. ${ }^{17}$ Assim, a solução adotada pelas especificações MLHIM, inspirada no MR menor da ISO 13606, foi definir um MM Minimalista.

Os princípios do MM minimalista, tal como definidos nas especificações MLHIM, são: (a) redução das classes e de atributos das classes de MR ao mínimo necessário para manter a estrutura do MM das especificações, mas ainda permitindo a persistência dos dados em aplicativos de qualquer tamanho e (b) a minimização do contexto semântico embutido no MR. Pela adoção dessa abordagem minimalista, os pacotes do MR MLHIM e as classes concretas utilizadas para a modelagem de conhecimento em CCDs foram definidas pelos conjuntos mínimos apresentado na Tabela 1.

Para fins de implementação, o MR MLHIM é formalmente expresso em XML Schemas (código fonte disponível em www.mlhim.org). Uma vez que um CCD é um conjunto de restrições às classes do MR MLHIM, expresso em XML Schema, para a descrição de um determinado conceito médico, demográfico ou administrativo, ele é uma definição portável de conceitos em saúde que pode ser trocado entre aplicativos baseados em MLHIM. Esta abordagem permite que estes aplicativos troquem informações semanticamente válidas, independentemente do projeto de banco de dados ou do mecanismo de persistência. ${ }^{18}$

Para o caso específico de aplicativos biomédicos e epidemiológicos de pequeno porte com propósitos específicos, uma implementação completa do MR MLHIM 
Tabela 1: Pacotes e classes concretas do MR MLHIM e suas funções na modelagem do conhecimento em saúde.

\begin{tabular}{|c|c|c|}
\hline Pacote MLHIM & Classe Concreta & Função \\
\hline \multirow[t]{3}{*}{ Content } & CareEntry ${ }^{\mathrm{a}}$ & $\begin{array}{l}\text { Define os atributos de protocolo and para todas as entradas rela- } \\
\text { cionadas à saúde dos indivíduos }\end{array}$ \\
\hline & AdminEntry ${ }^{\mathrm{a}}$ & Classe-filha de Entry para o registro de dados administrativos \\
\hline & Demographic Entry a & Classe-filha de Entry para o registro de dados demográficos \\
\hline \multirow[t]{3}{*}{ Structures } & Element ${ }^{\mathrm{b}}$ & $\begin{array}{l}\text { A variante terminal da classe Item, à qual se vincula uma instância } \\
\text { de uma das classes-filhas de DvAny do pacote Datatype }\end{array}$ \\
\hline & Cluster $^{b}$ & $\begin{array}{l}\text { A variante de agrupamento da classe Item, que pode conter instân- } \\
\text { cias adicionais de Item em uma lista ordenada }\end{array}$ \\
\hline & Slot $^{b}$ & $\begin{array}{l}\text { Uma estrutura que permite a inclusão de um ou mais CCDs dentro } \\
\text { de outro CCD }\end{array}$ \\
\hline
\end{tabular}

a. Classe-filha de Entry. b. Classe-filha de Item.

não é necessária. A conformidade com o MR MLHIM é obtida através da definição do modelo de dados do aplicativo, de acordo com a estrutura dos CCDs. Por exemplo, em um monitor de sinais vitais, os elementos de dados a serem persistidos devem seguir as estruturas correspondentes a um CCD, modelados de acordo com as exigências de persistência do aplicativo. Esta modalidade de desenvolvimento de software garante que os dados persistidos possam ser semanticamente interpretados por qualquer outro sistema baseado em MLHIM, desde que o mecanismo de persistência do aplicativo receptor baseie-se em uma implementação completa do MR MLHIM, e que os CCDs da aplicação remetente sejam partilhados entre ambos os aplicativos (por exemplo, por meio do HKCR).

Este processo define um ecossistema semanticamente interoperável de sistemas de informação para a área da saúde, onde aplicativos com propósitos específicos podem enviar dados válidos a partir do ponto de cuidado para aplicativos implementados em quaisquer outros serviços de saúde (incluindo PEHs). Neste tipo de ecossistema, o desenvolvimento eficiente de sistemas de apoio à decisão é mais viável, ${ }^{38}$ uma vez que todas as informações necessárias referentes a um determinado paciente podem ser recuperadas a partir de todos os serviços de saúde onde o paciente foi atendido.

\section{Conclusões}

A real vantagem da adoção de um MM minimalista, como proposto nas especificações MLHIM, é a oferta de uma grande variedade de aplicativos de saúde a serem desenvolvidos com base nos conceitos gerais definidas no MR. Especialistas do domínio que definem e criam seus CCDs podem colocá-los à disposição para o uso por múltiplos desenvolvedores, de modo que a estrutura dos dados não está aprisionada em um software específico, permitindo que a informação seja trocada entre vários aplicativos desenvolvidos de forma independente. Esta abordagem implementa corretamente a separação de papéis entre os desenvolvedores de aplicativos e os especialistas de domínio, cada um deles podendo otimizar sua contribuição para a evolução do ecossistema de e-saúde. 
Assim, a adoção aplicativos de saúde com propósitos específicos, baseados em MM, tem o potencial de apoiar iniciativas de e-saúde e, assim, atingir seus objetivos esperados de melhoria dos cuidados de saúde. Um caso de uso que pode ser mencionado é referente ao atendimento pré-hospitalar. Em geral, os casos que requerem atendimento pré-hospitalar recebem os primeiros socorros e necessitam de atendimento de maior complexidade em uma unidade hospitalar. Após a alta, o paciente continuará seu acompanhamento em nível ambulatorial, e a atenção básica será feita na Unidade de Saúde da Família mais próxima do seu domicílio. Assim, temos quatro instâncias do sistema de saúde envolvidas no cuidado a um paciente, sendo que as informações registradas em cada uma delas é crítica para o atendimento efetivo subsequente nas demais. Entretanto, tanto no atendimento pré-hospitalar como na atenção básica, aplicativos de grande porte tais como PEHs não são adequados, e muito provavelmente dispositivos móveis serão utilizados para a coleta de informações nestes dois cenários de atenção. Assim, uma abordagem minimalista para o desenvolvimento de aplicativos nestes níveis é mais factível do que a modelagem multinível baseada em arquétipos, não apenas pelo menor porte dos aplicativos que podem ser desenvolvidos, mas também pela maior possibilidade de se atender às especificidades locais do sistema de informação em saúde, em função da autonomia de modelagem oferecida pelos CCDs, em comparação com a complexidade induzida pelo mapeamento ou especialização de arquétipos, ou pela adoção de uma camada adicional de templates, para o atendimento aos requisitos de interoperabilidade semântica.

O que se nota do exemplo apresentado é a necessidade de se levar em conta a multiplicidade de soluções a serem implementadas no ecossistema dos aplicativos de saúde. Assim, no ponto de cuidado, as medidas apropriadas poderão ser mais oportunamente adotadas, melhorando a relação de custo-efetividade das intervenções de saúde e, no nível de governança, áreas mais amplas podem ser monitoradas e regiões prioritárias podem ser identificadas para intervenções comunitárias.

\section{Referências}

1. Tuohy $\mathrm{CH}$. Understanding the dynamics of change in the health care arena. In: Tuohy CH. Accitental logics. Oxford: Oxford University Press; 1999. p. 3-34.

2. Harper K, Armelagos G. The changing disease-scape in the third epidemiological transition. Int J Environ Res Public Health. 2010; 7:675-97.

3. Hoot NR, Aronsky D. Systematic review of emergency department crowding: causes, effects, and solutions. Ann Emerg Med. 2008; 52:126-36.

4. Johnson PJ, Blewett LA, Ruggles S, Davern ME, King ML. Four decades of population health data: the integrated health interview series as an epidemiologic resource. Epidemiology. 2008;19:872-5.

5. Hudson DL, Cohen ME. Uncertainty and complexity in personal health records. In: Proceedings of the 2010 Annual International Conference of the IEEE Engineering in Medicine and Biology Society (EMBC); 2010 Aug 31-Sep 4; Buenos Aires, Argentina. Piscataway: IEEE; 2010. p. 6773-6.

6. Ludwick DA, Doucette J. Adopting electronic medical records in primary care: lessons learned from health information systems implementation experience in seven countries. Int J Med Inform. 2009;78:22-31.

7. Zywietz C. Communication and interoperability for serial comparison in continuous health care: the new challenges. Stud Health Technol Inform. 2004; 108:172-80.

8. Shalom E, Shahar Y, Taieb-Maimon M, Martins SB, Vaszar LT, Goldstein MK et al. Ability of expert physicians to structure clinical guidelines: reality versus perception. J Eval Clin Pract. 2009;15:1043-53.

9. Schuler T, Garde S, Heard S, Beale T. Towards automatically generating graphical user interfaces from openEHR archetypes. Stud Health Technol Inform. 2006;124:221-6.

10. Saleem JJ, Russ AL, Neddo A, Blades PT, Doebbeling BN, Foresman BH. Paper persistence, workarounds, and communication breakdowns in computerized consultation management. Int J Med Inform. 2011; 80:466-79.

11. McGinn CA, Grenier S, Duplantie J, Shaw N, Sicotte C, Mathieu L et al. Comparison of user groups' perspectives of barriers and facilitators to implementing electronic health records: a systematic review. BMC Med. 2011; 9:46. http://www.biomedcentral.com/1741-7015/9/46. Acesso: 03/05/2012.

12. Gomes ATA, Ziviani A, Procópio VU, Moreira VM, Correa BSPM, Teixeira IM. Desenvolvimento dirigido a modelos para aplicações em saúde. In: Anais do Workshop de Informática Médica (WIM) 2011; Natal: SBC; 2011. http://www.lbd. dcc.ufmg.br/colecoes/wim/2011/0019.pdf. Acesso: 01/11/2012.

13. Souza RXO, Olivera AA. Abordagens orientadas a modelos no desenvolvimento de software em saúde: contribuições e perspectivas. In: Anais do Workshop de Informática Médica (WIM) 2012; Curitiba: SBC; 2012. p. 1-4. http://www.lbd.dcc. ufmg.br/colecoes/wim/2012/001.pdf. Acesso: 01/11/2012.

14. Michelsen L, Pedersen SS, Tilma HB, Andersen SK. Comparing different approaches to two-level modelling of electronic health records. Stud Health Technol Inform. 2005; 116:113-8.

15. Beale T. Archetypes and the EHR. Stud Health Tech Inform. 2003;96:238-44.

16. Kalra D, Beale T, Heard S. The openEHR Foundation. Stud Health Technol Inform. 2005;115:153-73. 
17. International Standards Organization. ISO 13606-1:2008. Health informatics - Electronic health record communication - Part 1: Reference model. Geneva: International Standards Organization; 2008.

18. Cavalini LT, Cook TW. Sistemas de informação em saúde: a importância do software livre e da modelagem multinível. J Bras TelesSaúde. 2012;1:16-23.

19. Chen R, Klein G. The openEHR Java reference implementation project. Stud Health Technol Inform. 2007;129:58-62.

20. Atalag K, Yang HY, Tempero E, Warren J. Model driven development of clinical information sytems using openEHR. Stud Health Technol Inform. 2011;169:849-53.

21. Lang T. Advancing global health research through digital technology and sharing data. Science. 2011;331:714-7.

22. Fioravanti A, Fico G, Arredondo MT, Salvi D, Villalar JL. Integration of heterogeneous biomedical sensors into an ISO/IEEE 11073 compliant application. In: Proceedings of the 2010 Annual International Conference of the IEEE Engineering in Medicine and Biology Society (EMBC); 2010 Aug 31-Sep 4; Buenos Aires, Argentina. Piscataway: IEEE; 2010. p. 1049-52.

23. Mense A, Sauermann S, Gerbovic G, Frohner M, Pohn B, Bruckner R et al. Usage of international standards for integrating extramural monitoring and personal health device data into medical information infrastructure. Stud Health Technol Inform. 2010;160:550-3.

24. Martinez-Costa C, Menarguez-Tortosa M, Fernandez-Breis JT. An approach for the semantic interoperability of ISO EN 13606 and OpenEHR archetypes. J Biomed Inform. 2010;43:736-46

25. van Deursen A, Klint P, Visser J. Domain-specific languages: an annotated bibliography. ACM SIGPLAN Not. 2000;35:26-36.

26. Kashfi H. The intersection of clinical decision support and electronic health record: a literature review. In: Proceedings of the 2011 Federated Conference on Computer Science and Information Systems (FedCSIS); 2011 Sep 18-21; Szczecin, Poland. Piscataway: IEEE; 2011. p. 347-53
27. Arikan S. openEHR for practical people. http://serefarikan.com/2010/10/18/openehrfor-practical-people. Acesso: 06/06/2012.

28. Beale T, Heard S. Archetype Definition Language ADL 1.4. London: openEHR Foundation; 2008

29. Leslie H. Clinical Knowledge Repository requirements. http://omowizard.wordpress. com/tag/clinical-knowledge-governance. Acesso: 12/06/2012.

30. Grimshaw J, Russell I. Achieving health gain through clinical guidelines. I: Developing scientifically valid guidelines. Qual Health Care. 1993;2:243-8.

31. Anderson T. Introducing XML. Part one: Overview. http://www.twriting.com/xmlintro. php. Acesso: 06/06/2012.

32. Chen M. Factors affecting the adoption and diffusion of XML and Web services standards for E-business systems. Int J Hum Comput Stud. 1993;58:259-79.

33. Sokolowski R, Dudeck J. XML and its impact on content and structure in electronic health care documents. In: Proceedings of the AMIA Symposium; 1999 Nov 6-10; Washington, DC. Philadelphia: Hanley \& Belfus; 1999. p. 147-51.

34. Rinner C, Janzek-Hawlat S, Sibinovic S, Duftschmid G. Semantic validation of standardbased electronic health record documents with W3C XML schema. Methods Inf Med. 2010;49:271-80

35. Shah R, Kesan JP. An empirical examination of open standards development. In: Proceedings of the 41st Annual Hawaii International Conference on System Sciences; 2008 Jan 07-10; Waikoloa, Hawaii. Piscataway: IEEE; 2008. p. 212.

36. Eren A, Subasi A, Coskun O. A decision support system for telemedicine through the mobile telecommunications platform. J Med Syst. 2008;32:31-5.

37. Hagland M. M.D. mobility express: are you ready to facilitate total physician mobility? Healthc Inform. 2011;28:10-6. 38. Manning M, Aggarwal A, Gao K, Tucker-Kellogg G. Scaling the walls of discovery: using semantic metadata for integrative problem solving. Brief Bioinform. 2009;10:164-76. 